\title{
DermAll nanomedicine for allergen-specific immunotherapy
}

\author{
Edina Garaczi, MD, PhD ${ }^{\mathrm{a}, \mathrm{b}, \mathrm{c}}$, Kornélia Szabó, PhD ${ }^{\mathrm{a}, \mathrm{c}}$, László Francziszti, MSc ${ }^{\mathrm{a}, \mathrm{d}}$, \\ Zsolt Csiszovszki, PhD ${ }^{\text {b,c }}$, Orsolya Lörincz, MSc ${ }^{\text {, c }}$, Enikő R. Tőke, PhD ${ }^{\text {b,c }}$, \\ Levente Molnár, MSc ${ }^{\text {, c }}$, Tamás Bitai, MSc ${ }^{\text {, c }}$, Tamás Jánossy, MD, $\mathrm{PhD}^{\mathrm{e}}$, \\ Zsuzsanna Bata-Csörgő, MD, DSc ${ }^{\text {a }}$, Lajos Kemény, MD, DSc ${ }^{\mathrm{a}, \mathrm{d}, *}$, Julianna Lisziewicz, PhD ${ }^{\mathrm{b}, \mathrm{c}}$ \\ ${ }^{\mathrm{a}}$ Department of Dermatology and Allergology, University of Szeged, Szeged, Hungary \\ ${ }^{\mathrm{b}}$ Genetic Immunity, Budapest, Hungary \\ ${ }^{\mathrm{c}}$ McLean, VA, USA \\ ${ }^{\mathrm{d}}$ Dermatological Research Group of the Hungarian Academy of Sciences, University of Szeged, Szeged, Hungary \\ ${ }^{\mathrm{e}}$ Institute of Surgical Research, University of Szeged, Szeged, Hungary
}

Received 27 December 2012; accepted 27 May 2013

\begin{abstract}
Allergen-specific immunotherapy (ASIT) the only disease-modifying treatment for IgE-mediated allergies is characterized with long treatment duration and high risk of side effects. We investigated the safety, immunogenicity and efficacy of a novel ASIT, called DermAll, in an experimental allergic rhinitis model. We designed and characterized DermAll-OVA, a synthetic plasmid pDNA/PEIm nanomedicine expressing ovalbumin (OVA) as model allergen. DermAll-OVA was administered topically with DermaPrep device to target Langerhans cells. To detect the clinical efficacy of DermAll ASIT we quantified the nasal symptoms and characterized the immunomodulatory activity of DermAll ASIT by measuring cytokine secretion after OVA-stimulation of splenocytes and antibodies from the sera. In allergic mice DermAll ASIT was as safe as Placebo, balanced the allergen-induced pathogenic $\mathrm{T}_{\mathrm{H}} 2$-polarized immune responses, and decreased the clinical symptoms by $52 \%[32 \%, 70 \%]$ compared to Placebo. These studies suggest that DermAll ASIT is safe and should significantly improve the immunopathology and symptoms of allergic diseases.
\end{abstract}

From the Clinical Editor: A novel allergen-specific immunotherapy for IgE-mediated allergies is presented in this paper, using an experimental allergic rhinitis model and a synthetic plasmid pDNA/PEIm nanomedicine expressing ovalbumin as model allergen. Over $50 \%$ reduction of symptoms was found as the immune system's balance was favorably altered toward more $\mathrm{T}_{\mathrm{H}} 2$-polarized immune responses. (C) 2013 Elsevier Inc. All rights reserved.

Key words: Nanomedicine; Epicutaneous Immunotherapy; DermaVir

Allergy is a major healthcare problem today. According to World Health Organization, hundreds of millions of people in the world (10-30\% of the population) suffer from allergic rhinitis (AR). ${ }^{1,2}$ AR results from an IgE-mediated inflammation of the

Abbreviations: AR, allergic rhinitis; ASIT, allergen-specific immunotherapy; EPIT, epicutaneous immunotherapy; LCs, Langerhans cells; OVA, ovalbumin; PEIm, polyethylenimine-mannobiose; pDNA, plasmid DNA.

Disclosure of potential conflict of interest: Julianna Lisziewicz, Tamás Bitai, Zsolt Csiszovszki, Edina Garaczi, Orsolya Lőrincz, Levente Molnár and Enikő R Töke hold shares in Genetic Immunity (PWRV). The rest of the authors have declared no conflict of interest.

This work was supported by the Asbóth Oszkár grant (HIKC05) of the Hungarian National Office for Research and Technology.

*Corresponding author: Genetic Immunity Kft., H-1045 Budapest, Berlini u. 47-49, Hungary.

E-mail address: lisziewj@geneticimmunity.com (L. Kemény). nasal mucosa. Patients suffer from nasal obstruction, sneezing, watery rhinorrhea, nasal itching, itchy palate, red and watery eyes, and from nonphysical symptoms including sleeping disturbance and emotional problems such as feeling tired, miserable and irritable. ${ }^{3}$ AR is a risk factor for asthma, a lifelong chronic inflammatory disorder of the airways that affects 300 million people by impairing their quality of life and negatively impacting the socio-economic welfare of society. ${ }^{4,5}$

Currently, the management of AR and other IgE-mediated allergies involves allergen avoidance, pharmacotherapy and allergen-specific immunotherapy (ASIT). ${ }^{6-9}$ Pharmacotherapy is the State of Art, because it is safe, well tolerated, suppresses the symptoms and improves the quality of life. However, these drugs neither cure allergy nor protect from disease progression including the development of asthma. ASIT is the only disease-modifying treatment for AR. ${ }^{7,10,11}$ Currently, highly 
standardized allergen extracts are employed for ASIT containing a poorly characterized mixture of allergens with other proteins. ${ }^{7,12}$ These extracts are administered via different routes. Presently available ASITs involve weekly subcutaneous and sublingual administration of increasing doses of such allergen extracts over a period of several years. ${ }^{13,14}$ The percentage of allergic patients undergoing ASIT is low due to the long duration of the therapy and the risk of severe systemic or local allergic reactions associated with the administration of the same allergen causing the disease. ${ }^{15,16}$

Innovations in ASIT focused partly on alternative delivery of allergen extracts. In 1957, two groups reported significant treatment success with epicutaneous immunotherapy (EPIT). Pautrizel et al repeatedly deposited the allergen extract on a slightly rubbed epidermis and Blamoutier et al applied the allergen extract onto extensively scarified area of skin. ${ }^{17,18}$ Current EPIT involves adhesive tape stripping, other abrasive methods and penetration enhancers like an occlusive patch leading to sweat accumulation. ${ }^{19,20}$ The first randomized placebo controlled clinical trial of EPIT with grass-allergen extracts reported significantly stronger amelioration of AR symptom than Placebo. ${ }^{19}$ The next dose-escalation study confirmed the efficacy of this EPIT, but demonstrated a significant risk of systemic allergic reactions associated with the application of allergen extract to tape-stripped skin. ${ }^{21}$ Recently, EPIT has been investigated with allergen extract formulated in nanoparticles in animal models. ${ }^{22}$ Other investigators used intralymphatic infusion of allergen extract to deliver allergen to the lymph node and to induce $\mathrm{T}_{\mathrm{H}} 1$ polarized immune responses. ${ }^{23}$

There is a high unmet medical need for a safer and more effective ASIT to treat IgE-mediated allergic diseases. Here we describe the preclinical investigations of a novel nanotechnological product, called DermAll ASIT, developed for the treatment of patients with IgE-mediated allergies. DermAll has several new features compared to present ASIT: (i) instead of an allergen extract it contains a plasmid DNA (pDNA) expressing well characterized molecular allergens; (ii) the pDNA is formulated to a synthetic pathogen-like nanomedicine to support delivery and potent expression of the allergen in antigen presenting cells; (iii) the nanomedicine is administered topically with DermaPrep device to target DermAll to the draining lymph nodes and to induce $\mathrm{T}_{\mathrm{H}} 1$-polarized immune responses. ${ }^{24-26}$

Ovalbumin (OVA) is one of the major allergens of chicken egg white, and egg allergy is one of the most prevalent forms of food allergy in children. ${ }^{27}$ To mimic the human IgE-mediated allergic diseases in mice we selected the OVA-induced AR model. We sensitized mice with intraperitoneal injection of OVA protein emulsified in aluminium hydroxide gel to induce $\mathrm{T}_{\mathrm{H}} 2$ type immune responses and produce OVA-specific IgE. These sensitized mice were subsequently challenged by intranasal administration of OVA solution to provoke allergic symptoms such as sneezing and nasal rubbing. ${ }^{28}$ In this mouse model we tested the safety, immunogenicity and efficacy of DermAllOVA, a prototype DermAll nanomedicine made with pDNA expressing OVA as a molecular allergen.

We present here the proof of concept for DermAll ASIT. We found that DermAll-OVA treatment was as safe as Placebo and balanced $\mathrm{T}_{\mathrm{H}} 2 / \mathrm{T}_{\mathrm{H}} 1$ OVA-specific immune responses in allergic mice. This allergen-specific immunomodulating activity of DermAll ASIT reduced the clinical symptoms of IgE-mediated AR.

\section{Methods}

Animals

Female, 8-week-old BALB/c mice were used in the experiments (Charles River Laboratories, Germany). The mice were kept on normal diet; food and water were available ad libitum. Annual care and experimental procedures were carried out in accordance with the local guidelines of University of Szeged. The study protocols were approved by the Committee on the Ethics of Animal Experiments of the University of Szeged.

\section{Plasmid expressing ovalbumin (OVA) as a model allergen}

pc-OVA plasmid consists of a pcDNA3 backbone and an OVA-encoding insert driven by the CMV promoter, kindly provided by A. Heit. ${ }^{29}$ For DermAll-OVA manufacturing the plasmid DNA was amplified using a Qiagen EndoFree Plasmid GIGA kit, according to the manufacturer's protocol (Hilden, Germany). Rigorous quality control measurements were performed to analyze the properties of each pDNA batch. The purity was measured by determination of OD 260/280 ratio and the integrity by agarose gel electrophoresis to determine the ratio of nicked and supercoiled DNA content.

\section{Manufacturing DermAll-OVA and Placebo nanomedicine vaccine}

The preparation of DermAll-OVA nanomedicine was described previously for DermaVir. ${ }^{30}$ Briefly, $1 \mathrm{mg} / \mathrm{mL}$ solutions of pDNA were mixed with PEIm (13.6 mM solution of linear $22 \mathrm{kDa}$ polyethylenimine containing 3\% mannobiose) in the presence of 6 volume D-glucose solvent. The Placebo vaccine contained PEIm mixed with solvent.

\section{Particle size measurements}

Measurements were performed from samples containing $10 \mu \mathrm{g} / \mathrm{mL}$ pDNA in DermAll-OVA (in triplicates), using Brookhaven ZetaPALS ${ }^{\mathrm{TM}}$ instrument equipped with quartz cuvette. Parameters: 3 runs, 30 seconds each; dust filter setting: 30; temperature: $25{ }^{\circ} \mathrm{C}$; angle: $90^{\circ}$; wavelength: $659.0 \mathrm{~nm}$; baseline: auto. All samples are diluted with ultra-pure water.

\section{Zeta potential analysis}

Zeta potential analysis measurements were performed using DermAll-OVA samples containing $10 \mu \mathrm{g} / \mathrm{mL}$ pDNA with Brookhaven ZetaPALSTM instrument equipped with "four side clear" plastic cuvette. Parameters: 5 runs, 10 cycles; temperature: $25^{\circ} \mathrm{C}$. All samples were diluted with ultra-pure water.

\section{Biological characterization of DermAll-OVA nanomedicine}

The in vitro expression test of the DermAll-OVA nanomedicine was performed by transfection of $293 \mathrm{~T}$ human cells. $16 \mu \mathrm{g}$ pDNA/DermAll-OVA was transfected into $5 \times 10^{6}$ cells in $5 \mathrm{~mL}$ DMEM culture medium (Invitrogen, DMEM, 1\% FBS, 1\% L-glutamine, 1\% Penicillin-Streptomycin) and incubated for 
A
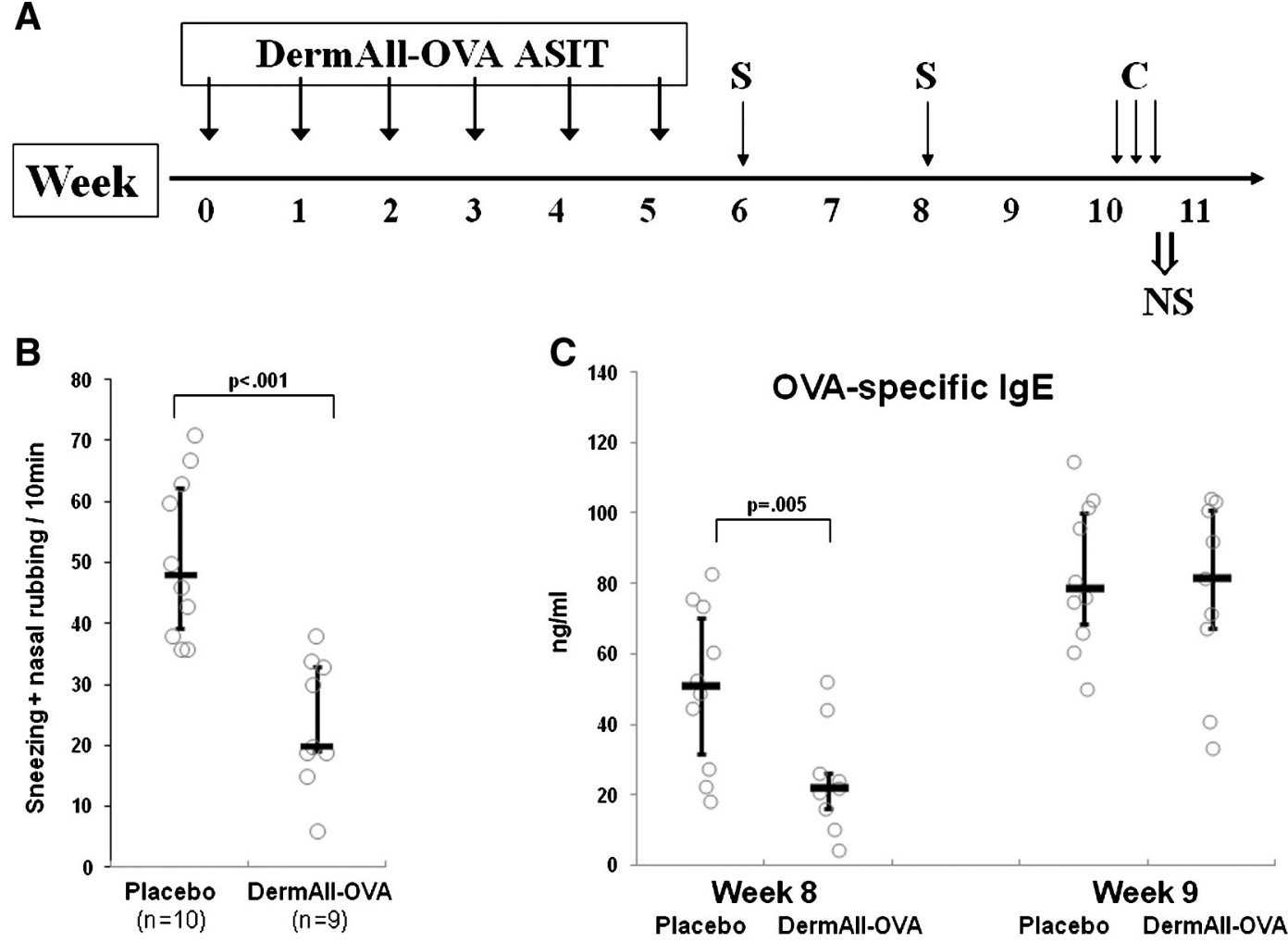

Figure 1. DermAll ASIT prevented acute allergic symptoms. (A) Experimental design: mice were treated with DermAll-OVA or Placebo six times weekly (DermAll-OVA ASIT), then sensitized (S) and subsequently challenged (C) with OVA. (B) DermAll-OVA reduced nasal symptoms (NS) compared to Placebo. (C) OVA-specific IgE levels. Data are presented as median and interquartile range.

48 hours at $37{ }^{\circ} \mathrm{C}$ in $5 \% \mathrm{CO}_{2}$ thermostat. To analyze both the secreted and intracellular OVA expression supernatants and cells were harvested. Cells were lysed in $1 \mathrm{~mL}$ lysis buffer (1\% SDS, $150 \mathrm{mM}$ Tris $\mathrm{pH}: 7.5$ ) and Western blot was performed. $1 \mu \mathrm{L}$ of the collected supernatant/cell lysate was analyzed on $10 \%$ polyacrylamide SDS-gel. Ovalbumin mouse anti-chicken monoclonal antibody was used to detect specifically the ovalbumin antigen (Lifespan Biosciences Inc., cat.: LS-C47187).

\section{Topical DermAll-OVA administration (DermaPrep)}

Mice were anesthetized and their fur was removed from the back by shaving. Then, the skin was prepared by rubbing the skin 20 times with a sponge adopted from DermaPrep device followed by tape stripping. ${ }^{26}$ Total volume of $200 \mu \mathrm{L}$ DermAll-OVA (containing $25 \mu \mathrm{g}$ pDNA) or Placebo was applied on approximately $20 \mathrm{~cm}^{2}$ area on the prepared skin of each mouse. The selected dose of pDNA was based on our previous monkey studies, where $25 \mu \mathrm{g}$ pDNA containing nanomedicine was administered on four different skin sites. ${ }^{31}$ This immunization procedure has been standardized to ensure the reproducibility, and avoid serious inflammation and tissue damage.

\section{Safety parameters of DermAll-OVA immunization}

To assess the safety of DermAll ASIT body weight and local adverse reactions at the treatment sites were measured. The body weights were measured weekly for each mouse during every preventive and therapeutic studies. The treatment skin site of
DermAll-OVA was investigated before and 24 hours after each immunization. The gross pathology findings were recorded after mice were sacrificed.

\section{DermAll-OVA placebo controlled and blinded immunization protocols}

Naïve mice were immunized six times weekly with DermAllOVA or Placebo (Figure 1,A). At weeks 6 and 8 mice were sensitized and at week 10 challenged with OVA for three consecutive days. Consequently, a blinded operator evaluated the nasal symptoms (see methods later). At the end of the study protocol (week 11) mice were sacrificed and splenocytes were collected.

\section{Sensitization with OVA protein}

$25 \mu \mathrm{g} /$ mouse OVA (grade III. Sigma-Aldrich) diluted by sterile phosphate buffered saline (PBS) containing $1 \mathrm{mg} / \mathrm{mouse}$ aluminium hydroxide gel (Sigma-Aldrich). $300 \mu \mathrm{L}$ of this solution was injected intraperitoneal to unanesthetized mice.

\section{Nasal challenge with OVA protein}

$500 \mu \mathrm{g} /$ mouse OVA was diluted in $10 \mu \mathrm{L}$ PBS. $5 \mu \mathrm{L}$ of this solution was administered on each nasal cavity of the mouse for three consecutive days.

\section{Quantitative assessment of clinical symptoms}

On the third day of challenge nasal symptoms were evaluated by counting the number of sneezing and nasal rubbing for 
A

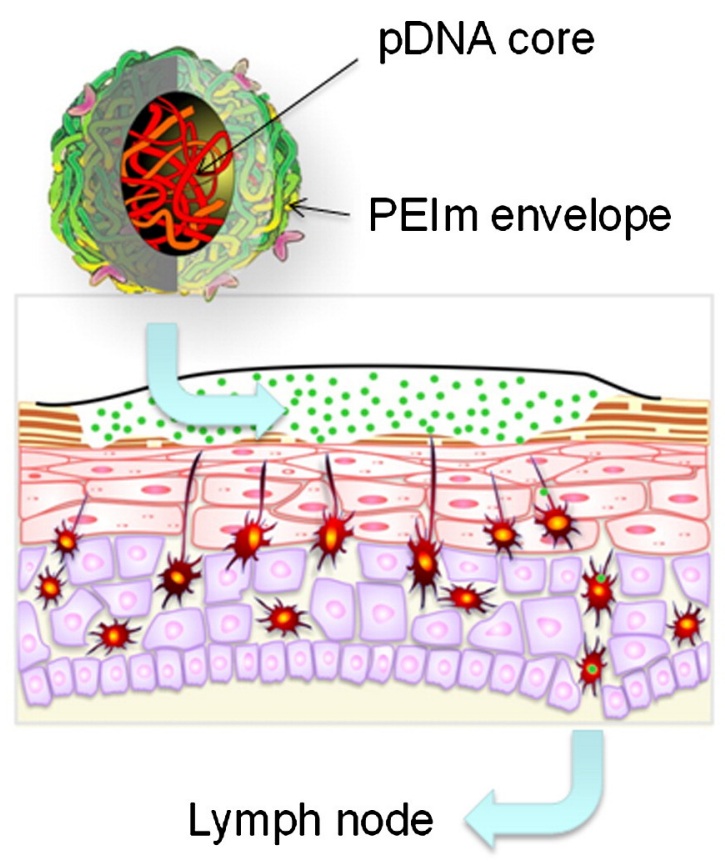

B

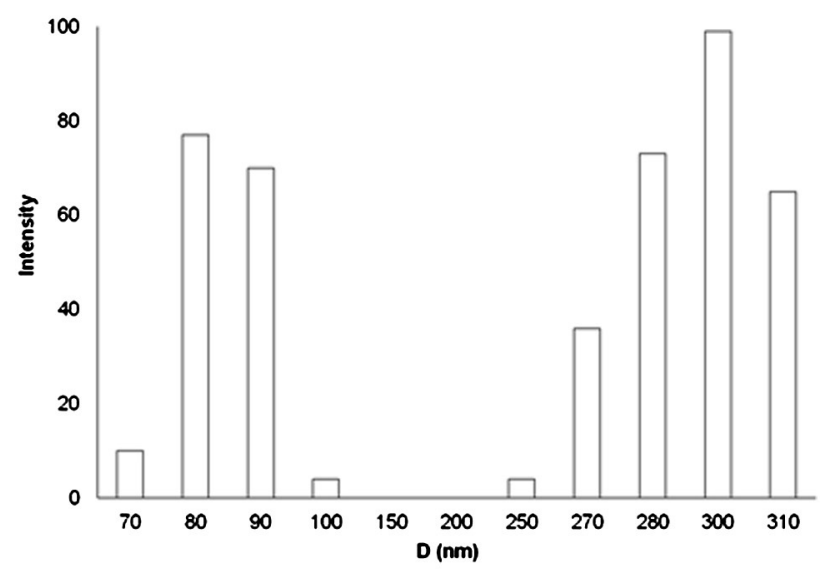

C

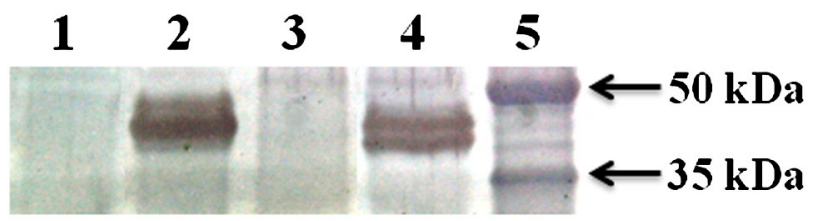

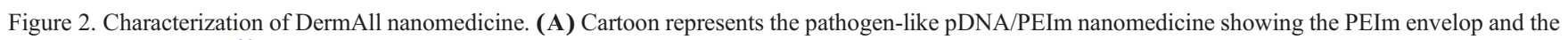

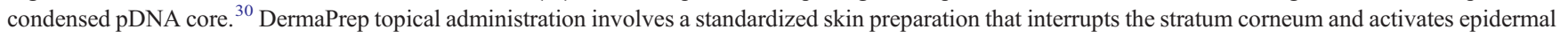

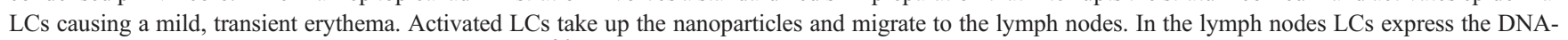

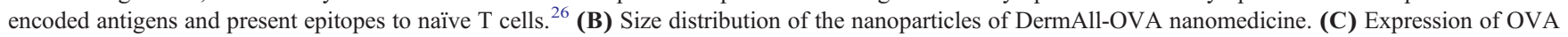

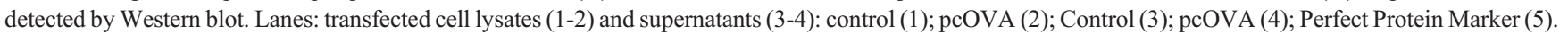

10 min after OVA intranasal provocation for each mouse. Counting of the symptoms was performed by a blinded operator.

\section{Detection of OVA-specific $\operatorname{IgE}$, IgG1 and $\operatorname{IgG} 2 a$ in serum}

Serum samples were collected and sera were kept frozen at $20{ }^{\circ} \mathrm{C}$ until analysis. OVA-specific IgE, IgG1 and IgG2a levels were analyzed with ELISA Quantitation Kit according to the manufacturer's instruction (Bethyl Laboratories). Briefly, 96well microplate was coated with $5 \mu \mathrm{g} /$ well ovalbumin (grade $\mathrm{V}$., Sigma-Aldrich) dissolved in normal saline and incubated overnight at $4{ }^{\circ} \mathrm{C}$. The plate was then washed then blocked with $1 \%$ bovine serum albumin at $37^{\circ} \mathrm{C}$ for 1 hour. Serum samples were diluted (1:5 for IgE; 1:10 for IgG1 and IgG2a) and added into the wells and incubated for 1 hour. After washing, horseradish peroxidase conjugate solution (1:20,000 dilutions) was added and incubated for 1 hour. Enzymatic activity was measured with enzyme substrate solution (equal volumes of Tetramethylbenzidine (TMB) peroxidase substrate and peroxidase solution) for $30 \mathrm{~min}$ at $37^{\circ} \mathrm{C}$. The absorbance was measured at 450 and $620 \mathrm{~nm}$ using an ELISA plate reader (Thermo, Elektroscan).

\section{Cytokine assays}

$4 \times 10^{6}$ splenocytes were plated in 6-well plates in $2 \mathrm{~mL}$ RPMI 1640 (GIBCO, Life Technologies) supplemented with $10 \%$ heat-inactivated Fetal Bovine Serum (Thermo Scientific
Hyclone), 5\% penicillin/streptomycin, 5\% L-Glutamine and 0,1\% $50 \mathrm{mM}$ Mercapto-ethanol (Sigma-Aldrich). Splenocytes were activated with $50 \mu \mathrm{g} / \mathrm{mL}$ OVA (grade V., Sigma-Aldrich) or culture medium (assay control) and incubated at $37{ }^{\circ} \mathrm{C}$ in $\mathrm{CO}_{2}$ thermostat. After 72 hours, supernatants were collected and stored at $-20{ }^{\circ} \mathrm{C}$ until further analysis.

OptEIA Elisa Set was used to measure the levels of IL-4, IL5 , IL-10, and IFN- $\gamma$ according to the manufacturer's instructions (Becton Dickinson). Briefly, plates were coated overnight at $4{ }^{\circ} \mathrm{C}$ with the anti-mouse monoclonal antibody. The plates were washed then blocked with $10 \%$ fetal bovine serum for 1 hour. Samples and standards were added and incubated for 2 hours. Then plates were incubated with biotinylated anti-mouse monoclonal antibody and streptavidin-horseradish peroxidase conjugate of a 1:250 dilution for 1 hour. Antigen-antibody complexes were detected with a substrate solution (containing TMB and hydrogen peroxide). The absorbance was measured at 450 and $620 \mathrm{~nm}$ using an ELISA reader (Thermo, Elektroscan).

Data were calculated as the cytokine concentration in the supernatants of OVA-activated cells minus in assay controls. In the therapeutic immunization protocol assay controls were not deducted from OVA-activated samples.

\section{Statistical analyses}

Statistical analyses were performed by using SAS 9.2 (SAS Institute Inc., Cary, NC, USA). Nasal symptoms were analyzed 

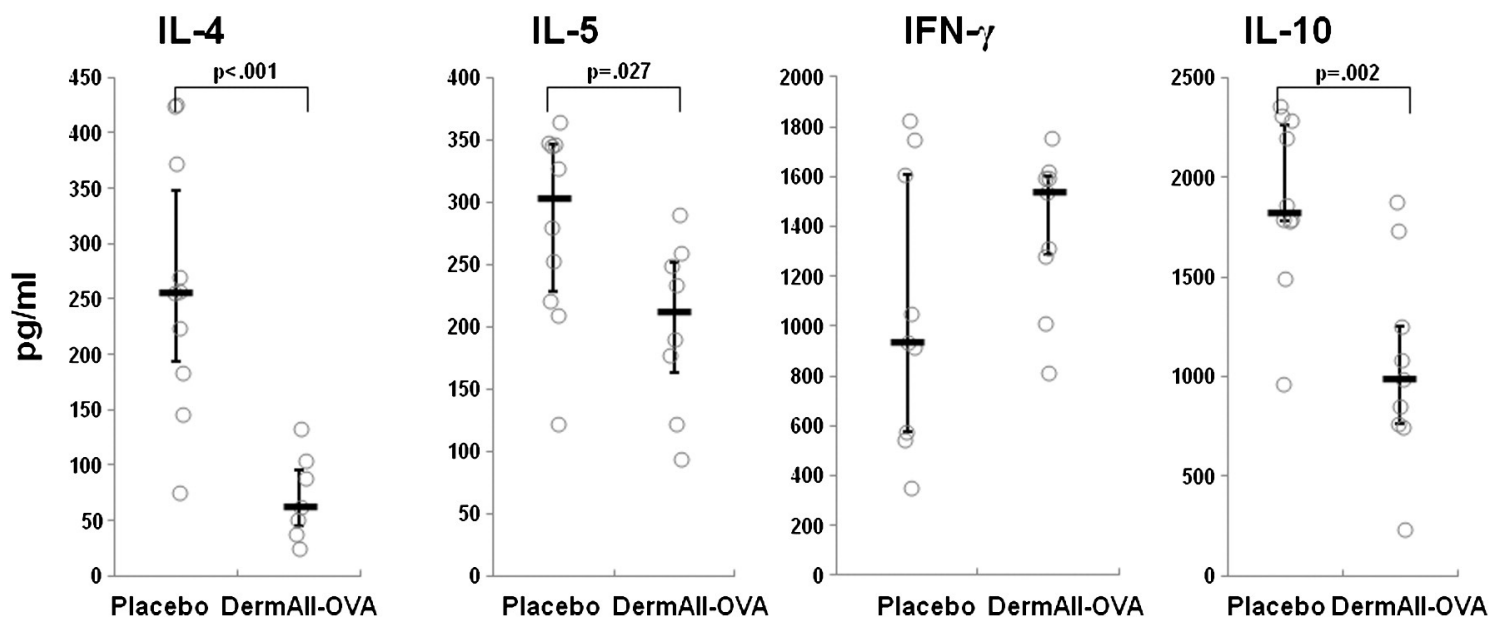

Figure 3. Allergen-specific immune responses are balanced after DermAll ASIT. Mice were treated with DermAll-OVA ASIT, subsequently sensitized and challenged with OVA (see Figure 2, A). OVA-specific IL-4, IL-5, IFN $\gamma$ and IL-10 responses are presented as median and interquartile range.

with Wilcoxon rank sum test for the preventive immunization protocols and with Hollander's test for the therapeutic protocol. For estimating treatment effects we used Hodges-Lehmann point estimate with exact $95 \%$ confidence interval on the logtransformed data. All other comparisons were made with Wilcoxon rank sum test. Cytokine data are presented in median and quartiles (Q1-Q3). In all cases, $P$ values less than 0.05 were considered statistically significant.

\section{Results}

\section{Characterization of DermAll-OVA nanomedicine}

We constructed and characterized DermAll-OVA pathogenlike nanomedicine consisting of a synthetic mannosylated polyethylenimine (PEIm) "envelop" and an OVA encoding pDNA "core" (Figure 2, A). The zeta potential of DermAllOVA was $+8.6 \pm 6.9 \mathrm{mV}$ demonstrating that the cationic PEIm totally encapsulated the condensed anionic pDNA. The mean effective diameter of the DermAll-OVA nanomedicine was $141 \pm 18 \mathrm{~nm}$, with a bimodal size distribution containing two discrete size ranges of nanoparticles (Figure 2, $B$ ). We described earlier that the shape and structure of the two nanoparticle populations were identical and their biological functions were the same ${ }^{30}$ These nanoparticles have the appropriate size for receptor mediated entry to Langerhans cells as they are in the virus size range of 50-500 $\mathrm{nm}$.

We investigated the expression of OVA after transfecting 293 T human cells with DermAll-OVA nanomedicine. OVA expression was detected by Western blot as a $45 \mathrm{kDa}$ protein both intracellularly and in the supernatant confirming that OVA was secreted and had the attached carbohydrate chain required for correct protein folding in the cells (Figure 2, C) ${ }^{32}$

DermaPrep medical device (CE-marked) utilizes a topical immunization procedure developed for LCs targeting nanomedicine administration in human subjects. ${ }^{26}$ This procedure was adopted in our mice study to provide clinically relevant safety, immunogenicity and efficacy results. The maximum dose pDNA $(25 \mu \mathrm{g})$ that corresponds to $5 \times 10^{12}$ nanoparticles was administered topically per mice. The novel mechanism of action of a related pDNA/PEIm nanomedicine (DermaVir) has been previously characterized in mice, macaques, rabbits and human subjects (Figure $2, A){ }^{25}$

\section{DermAll ASIT prevents the induction of allergic symptoms}

To assess the preclinical efficacy of DermAll ASIT we developed an animal model relevant to human AR. During our preliminary experiments we have worked out a sensitization and challenge protocol in mice that resulted in nasal symptoms similar to the clinical symptoms of AR in human subject. We quantified these nasal symptoms by counting the sneezing and nasal rubbing for ten minutes after allergen challenge. We interpreted the reduced quantity of rubbing and sneezing after DermAll ASIT as a clinically relevant improvement of AR symptoms.

In our previous experiments, we found that repeated DermAllOVA immunizations three times biweekly were not sufficient to prevent allergic sensitization in naïve mice (data not shown). Next we treated naïve mice weekly with DermAll-OVA and Placebo for six times. One week after the final immunization, mice were sensitized and subsequently challenged with OVA and the nasal symptoms were quantified (Figure 1, $A$ ). The median of sneezing and nasal rubbing in the Placebo group was 48 (38-63). In contrast, mice treated with DermAll-OVA ASIT had significantly less 20 $(19-33)$ nasal symptoms $(P<0.001)$ (Figure $1, B)$. Our results suggested the disease-modifying property of DermAll ASIT, characterized by $52 \%[32 \%, 70 \%]$ reduction of the AR symptoms.

Conventional ASITs induce a transient increase in $\operatorname{IgE}$ levels followed by a gradual decrease over months and years of treatment. ${ }^{33,34}$ However, it was also shown that the level of allergen-specific IgE did not correlate with the clinical symptoms in allergic patients. ${ }^{35} \mathrm{We}$ found that DermAllOVA ASIT decreased by $55 \%$ [12\%, 75\%] the OVA-specific IgE production after the first sensitization (week 8). IgE levels were $22 \mathrm{ng} / \mathrm{mL}(16-26)$ in the DermAll-OVA group and 51 (27-73) in the Placebo group $(P=0.005)$ (Figure $1, C)$. One week after the second sensitization (week 9) the median $\operatorname{IgE}$ levels increased to $81 \mathrm{ng} / \mathrm{mL}$ (67-101) in the DermAll-OVA group and 78 (66-101) in the Placebo group $(P=0.842)$. The 

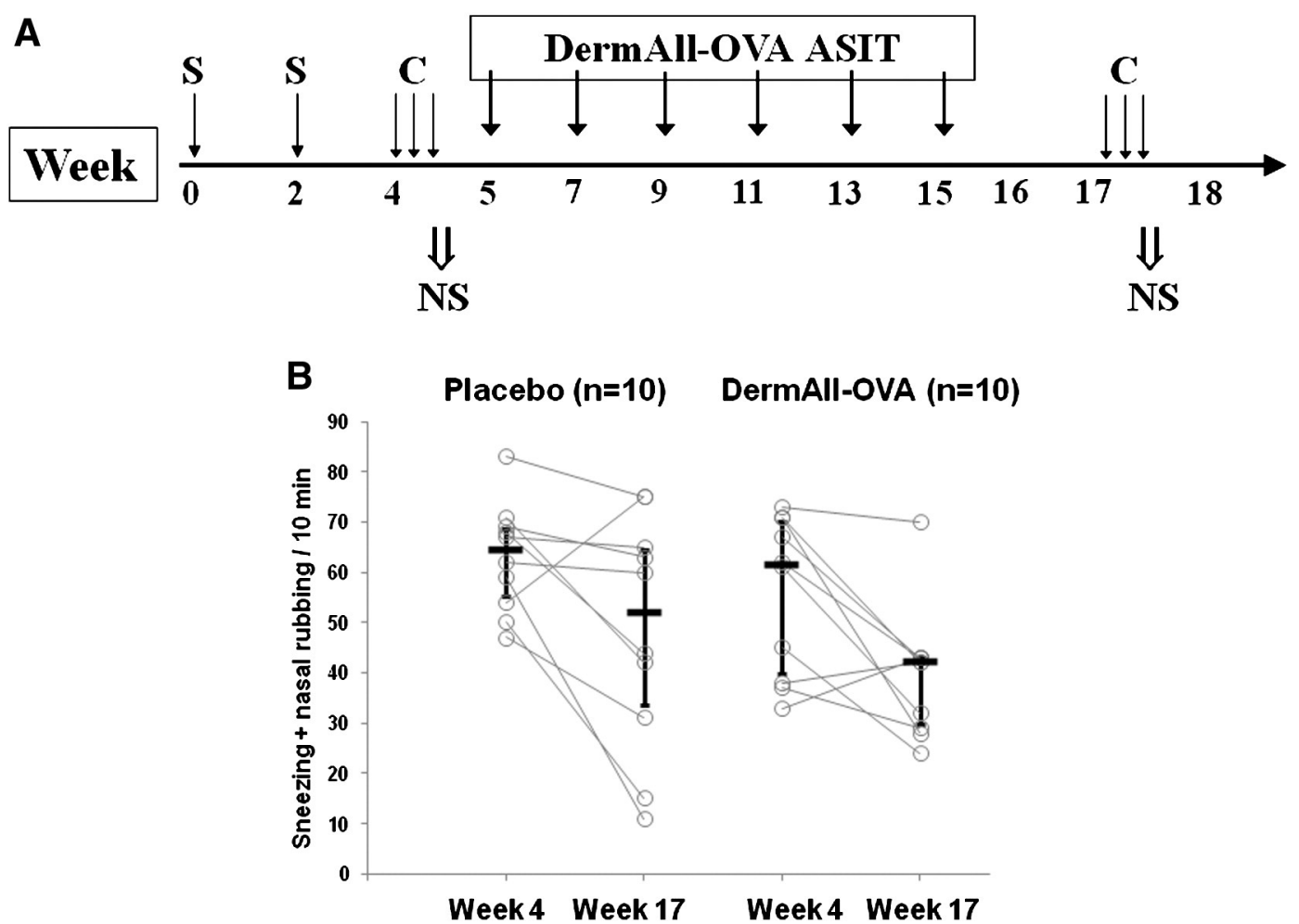

Figure 4. DermAll ASIT in allergic mice. (A) Experimental design: Mice were sensitized (S) two times and subsequently challenged (C) with OVA. These allergic mice were treated six times biweekly with DermAll-OVA or Placebo. Two weeks later mice were challenged again and nasal symptoms (NS) were quantified. (B) Nasal symptoms before and after DermAll-OVA and Placebo ASIT. $P=0.625$ was calculated by Hollander's test.

decreased OVA-specific IgE production at the initial part of the sensitization suggested that DermAll ASIT could modulate the allergen-induced $\operatorname{IgE}$ responses.

The levels of OVA-specific IgG1 and IgG2a between the 2 groups were found to be comparable during the sensitization at week 8 and 9 (data not shown).

To study whether the weekly DermAll-OVA administration schedule was essential for the clinical benefit we doubled the time between immunizations. Naïve mice were immunized 6 times biweekly with DermAll-OVA and Placebo. Four weeks after the final immunization, mice were sensitized and subsequently challenged with OVA. In this experiment the median number of AR symptoms was 96 (89-103) in the Placebo and 58 (52-76) in the DermAll-OVA group $(P<0.001)$ demonstrating a statistically significant treatment effect, which can be quantified by a $36 \%$ [20\%, 49\%] reduction of the AR symptoms (see Figure in Supplementary Materials). This result confirmed the disease modifying property of DermAll-OVA nanomedicine.

\section{DermAll ASIT prevents pathogenic $T_{H} 2$-polarized $T$ cell responses to allergen exposure}

Presently available conventional ASITs are associated with an immune deviation in favor of $\mathrm{T}_{\mathrm{H}} 1$ responses with an overproduction of IFN $\gamma .{ }^{11,13} \mathrm{We}$ hypothesized that DermAll ASIT would balance allergen-specific $\mathrm{T}$ cell responses resulting in the modification of the allergic symptoms. To determine the immune modulating effect of DermAll-OVA we measured the OVA- specific $\mathrm{T}$ cell responses after sacrificing the mice (week 11) in the experiment depicted in Figure 1, $A$.

To assess the $\mathrm{T}_{\mathrm{H}} 2$ responses we measured both IL-4 and IL-5. The OVA-specific IL-4 responses in the DermAll-OVA and the Placebo groups were $63 \mathrm{pg} / \mathrm{mL}$ (38-104) and $256 \mathrm{pg} / \mathrm{mL}$ (184$373)$, respectively $(P=0.0004)$ (Figure 3$)$. The OVA-specific IL-5 responses were $212 \mathrm{pg} / \mathrm{mL}(150-254)$ in the DermAllOVA, and $303 \mathrm{pg} / \mathrm{mL}(220-347)$ in the Placebo group $(P=$ 0.027) (Figure 3). These data showed that DermAll-OVA induced a $75 \%[51 \%, 86 \%]$ decrease in IL-4 and a $28 \%[0 \%$, $49 \%$ ] decrease in IL-5 secretion and suggested that DermAllOVA prevented the development of pathogenic allergen-induced $\mathrm{T}_{\mathrm{H}}$ 2-type immune responses.

To assess the $T_{H} 1$ responses we measured IFN $\gamma$ levels. The median of OVA-specific IFN $\gamma$ responses was $1,541 \mathrm{pg} /$ $\mathrm{mL}(1,286-1,599)$ in the DermAll-OVA group and 939 (577$1,610)$ in the Placebo group $(P=0.297)$ (Figure 3$)$. These results demonstrated that the prevention of allergen-induced $\mathrm{T}_{\mathrm{H}}$ 2-polarized immune response by DermAll ASIT did not cause a $\mathrm{T}_{\mathrm{H}} 1$-type pathogenic immune activation and suggested that DermAll ASIT balanced the allergen-specific $\mathrm{T}$ cell responses.

To assess the immunomodulation we measured the pleiotropic cytokine IL-10. The OVA-specific IL-10 responses in the DermAll-OVA and Placebo control groups were $989 \mathrm{pg} / \mathrm{mL}$ $(765-1,250)$ and $1,825 \mathrm{pg} / \mathrm{mL}(1,778.6-2,287)$, respectively $(P=0.002)$ (Figure 3$)$. Our results showed that DermAll-OVA led to a significant reduction of IL-10 production in allergic mice. 

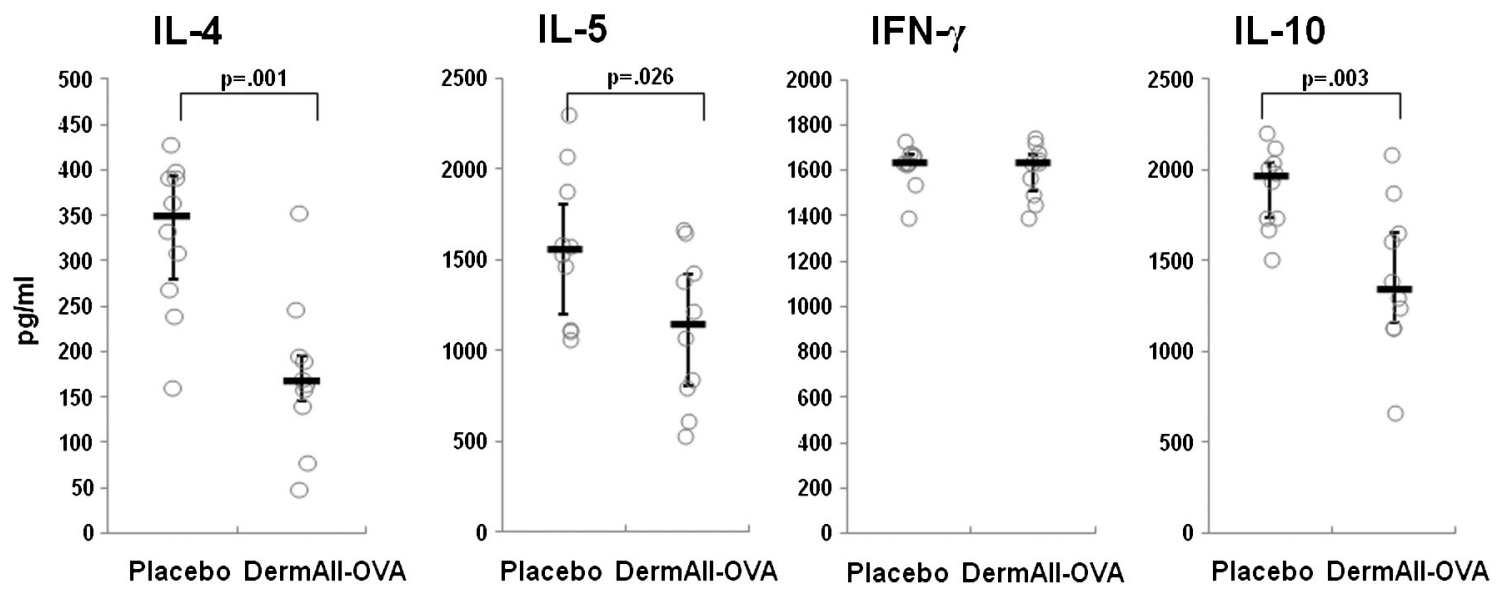

Figure 5. DermAll ASIT facilitates the immunological recovery from allergic response. Allergic mice were treated with DermAll-OVA and Placebo ASIT and challenged with OVA (see Figure 4, A). OVA-specific IL-4, IL-5, IFN $\gamma$ and IL-10 responses are presented as median and interquartile range.

Efficacy and immunomodulatory activity of DermAll ASIT during acute allergic symptoms

Next, we evaluated the efficacy of DermAll ASIT during acute allergic symptoms. OVA allergy was induced in mice with sensitization and challenge as depicted in Figure 4, A. These highly symptomatic allergic mice were treated six times biweekly either with DermAll-OVA or Placebo. Two weeks after the final immunization mice were challenged again to measure the treatment effect of DermAll-OVA.

The limitation of this animal model was that most of the mice treated with Placebo recovered after 12 weeks without allergen exposure. Within these 12 weeks of recovery the median sneezing and nasal rubbing in the Placebo group decreased $20 \%$ from 64.5 (54-69) to 52 (31-65) (Figure 4, B). Similarly, in the DermAll-OVA group the nasal symptoms decreased $32 \%$ from 61.5 (38-71) to 42 (29-43) (Figure 4, B). The speed of recovery of AR symptoms measured between DermAll-OVA and Placebo ASITs was statistically not significant $(P=0.625)$. Repeated experiments showed similar results. These results suggested that DermAll ASIT administered during acute allergy might cause a faster recovery of AR symptom but our results did not prove the treatment efficacy.

To investigate the immunomodulatory effect of DermAll-OVA ASIT on symptomatic mice we measured the OVA-specific $T$ cell cytokine responses. Interestingly, we found that the $\mathrm{T}_{\mathrm{H}} 2$-type cytokine production was significantly lower in allergic mice treated with DermAll-OVA compared to Placebo. The OVAspecific IL-4 responses in the DermAll-OVA and Placebo control groups were $168 \mathrm{pg} / \mathrm{mL}$ (141-196) and 349 (270-393), respectively $(P=0.001)$ (Figure 5$)$. The median of OVA-specific IL-5 responses was $1,147 \mathrm{pg} / \mathrm{mL}(798-1,431)$ in the DermAll-OVA group and $1,555 \mathrm{pg} / \mathrm{mL}(1,119-1,877)$ in the Placebo control group $(P=0.026)$ (Figure 5). These results suggested that DermAll treatment facilitated the immunological recovery from AR.

We measured the levels of a $T_{H} 1$-type cytokine, IFN $\gamma$. The OVA-specific IFN $\gamma$ responses in the DermAll-OVA and the Placebo groups were 1,636 pg/mL (1,493-1,679), and 1,633 pg/ $\mathrm{mL}(1,627-1,674)$, respectively $(P=0.985)$ (Figure 5$)$. These results confirmed that DermAll ASIT treatment results in balanced allergen-specific immune responses.

We measured the secretion of IL-10. We found that the OVAspecific IL-10 responses were $1,349 \mathrm{pg} / \mathrm{mL}(1,134-1,663)$ in the DermAll-OVA group, and $1,969 \mathrm{pg} / \mathrm{mL}(1,739-2046)$ in the Placebo control group $(P=0.002)$ (Figure 5). These results confirmed that DermAll ASIT led to a significant reduction in IL-10 secretion in allergic mice.

\section{Safety features of DermAll-OVA immunization}

Current ASITs performed with allergen extracts are associated with the risk of severe allergic reactions. ${ }^{15,16}$ Specifically, EPIT of allergic patients with allergen extracts induced eczema at the vaccine application site and was associated with systemic allergic reactions. ${ }^{19,21}$ Therefore, one of the main objectives in DermAll development was to improve the safety of the presently available ASITs.

The body weights were measured weekly for each mouse during every preventive (Figure 1) and therapeutic (Figure 4) study. We found that DermAll ASIT did not cause weight loss in mice. No abnormalities were seen during necropsy after external, abdominal and thoracic examinations in DermAllOVA immunized mice (data not shown). These results showed the safety features of DermAll-OVA and suggested that DermAll ASIT would not cause systemic side effects in human subjects.

To determine the local skin reactogenicity (any adverse reaction on the skin), the treatment skin site of DermAll-OVA was investigated before and 24 hours after each immunization procedure. No erythema, oedema, eczema or other long-lasting skin reactions were observed after repeated DermAll immunizations to highly allergic mice (Figure 4). There were no differences in local skin reactogenicity between DermAll and Placebo groups in naïve mice either (Figure 1). Local, transient skin irritation seen immediately after DermAll application was due to the DermaPrep skin preparation (rubbing and tape stripping) in both groups. The same results were found with DermaVir HIV-specific immunotherapy during both preclinical 


\section{Immunological and Clinical Benefits}

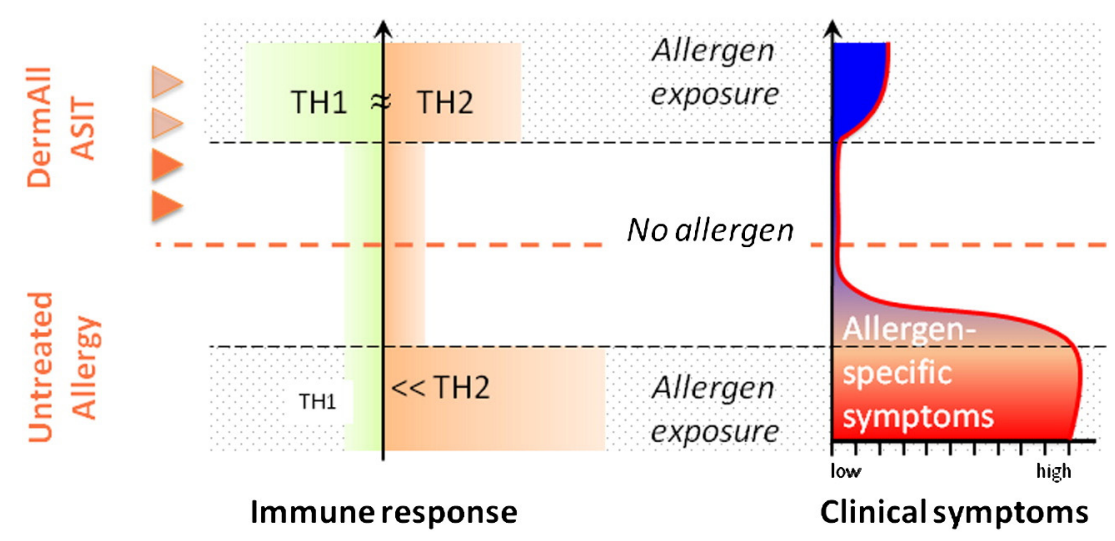

Figure 6. Potential disease modifying activity of DermAll ASIT. Allergen exposures cause pathogenic $\mathrm{T}_{\mathrm{H}}$ 2-polarized immune responses. We hypothesize that allergy might be cured by balancing the $\mathrm{T}_{\mathrm{H}} 2 / \mathrm{T}_{\mathrm{H}} 1$ responses to allergens. The immunological activity of DermAll was associated with clinical improvement measured as the reduction of allergic symptoms. Based on the results in mouse model we propose that DermAll might be most effective when administered in an allergen-free period to increase the immunological and clinical recovery during the following allergen exposures. However, we expect a faster recovery in the absence of safety problems when we continue DermAll during the period of allergen exposures to keep balancing the allergen-specific immune responses.

and clinical studies. ${ }^{25}$ These results suggested that DermAll ASIT might have excellent safety features in allergic human subjects.

\section{Discussion}

We introduced here DermAll, a novel ASIT, developed to improve both the safety and the efficacy of presently available allergen extract-based ASITs. DermAll ASIT is a needle-free patch treatment with a nanomedicine containing an allergenencoding pDNA. DermAll ASIT has two components: a synthetic pDNA/PEIm nanomedicine for LC-targeted nuclear delivery of pDNA to express epitopes in the lymph nodes, and a medical device (DermaPrep) for topical administration of the nanomedicine. The topical administration delivers the pathogenlike nanomedicine into the epidermis to target LCs. These professional antigen presenting cells, specialized for uptake of pathogens, pick up the nanoparticles and migrate to the draining lymph nodes. ${ }^{24-26}$ Allergen exposure induces IgE production in plasma cells and an increased $\mathrm{T}_{\mathrm{H}}$ 2-type cytokine production in lymphoid tissues and nasal mucosa. ${ }^{13}$ We hypothesized that the allergen-specific $\mathrm{T}_{\mathrm{H}}$ 1-type immune response induced by DermAll ASIT balances the immune deviation caused by the allergens and decreases the nasal symptoms of AR.

DermAll is the pipeline product of DermaVir HIV-specific immunotherapy that was feasible and well tolerated in almost 100 HIV-infected subjects. ${ }^{24-26}$ Three clinical trials showed that DermaVir therapy was as safe as Placebo and induced robust antigen-specific $T_{H} 1$ polarized immune responses. We found that DermAll ASIT was safe; it showed no systemic side effects and did not induce pathogenic immune responses. The local skin reactogenicity of DermAll ASIT was comparable to Placebo in both naïve and allergic mice. Similar safety and immunogenicity profile was established in animal models and human subjects with DermaVir HIV-specific immunotherapy. The human data with DermaVir validate the immunogenicity of DermAll obtained from mouse model; however the safety and efficacy should be tested in allergic patients.

DermaVir and DermAll are the same pDNA/PEIm nanomedicines, the same chemical entities, and the only difference between them is the sequence of the pDNA that ensures the antigen specificity of the nanomedicine. Both DermaVir and DermAll are administered topically to target the pDNA-encoded antigens to LCs. ${ }^{24}$ These nanomedicine-containing LCs migrate to the lymph nodes, express the antigens and present the epitopes to naïve T cells (Figure 2). We showed potent in vitro expression of OVA from the DermAll nanomedicine confirming previous results obtained with pcOVA plasmid in vivo tested in mice. ${ }^{29}$ We have previously demonstrated nanomedicine-derived gene expression in the draining lymph nodes after topical administration of a pDNA/PEIm nanomedicine in BALB/c and C57BL/6 mice and macaques. ${ }^{24}$ Antigen expressing LCs were mainly located in the paracortical area of the lymph node, which is also referred to as the $\mathrm{T}$ cell area.

We demonstrated that DermAll ASIT prevented the induction of clinical symptoms of AR in a mouse model. We found that at least six repeated DermAll-OVA treatments were required to prevent the clinical symptoms. These repeated DermAll immunizations administered either weekly or biweekly significantly protected the animals from the development of allergic symptoms (Figure 1). In contrast, we could not demonstrate statistically significant clinical benefit when DermAll ASIT was used to treat highly allergic, symptomatic animals (Figure 4). Unfortunately, longer treatment period of allergic mice also failed to demonstrate clinical efficacy, since even in the absence of any treatment mice recovered within 12 weeks. Most importantly, DermAll ASIT prevented $\mathrm{T}_{\mathrm{H}} 2$ polarized allergic immune responses and also facilitated the immunological recovery in mice with acute allergic symptoms. Based on these results we propose that repeated DermAll ASIT would be a safe and effective disease modifying treatment for allergic patients. 
DermAll ASIT has a novel mechanism of action to balance allergen-specific immune responses. DermAll-OVA immunization decreased the $\mathrm{T}_{\mathrm{H}}$ 2-type immune responses (IL-4 and IL-5) both in subsequently sensitized (Figure 3) and in allergic mice (Figure 5), and promoted a shift of the immune response to a balanced $\mathrm{T}_{\mathrm{H}} 2 / \mathrm{T}_{\mathrm{H}} 1$ profile.

Conventional ASITs induce a transient increase in specific IgE levels followed by a gradual decrease over months or years of treatment. However, it is also known that the level of specific $\mathrm{IgE}$ does not correlate directly with the clinical symptoms in allergic patients. ${ }^{35}$ We found that DermAll immunization did not induce the production of OVA-specific $\operatorname{IgE}, \operatorname{IgG} 1$ or IgG2a in naïve mice (data not shown). The decreased OVA-specific IgE production during the sensitization in preventively immunized mice suggested that DermAll ASIT could modulate the allergeninduced IgE responses (Figure 1).

Our proposed treatment of allergic patients with DermAll ASIT is summarized in Figure 6. Repeated allergen exposures induce pathogenic type 2 cytokine responses and allergic symptoms in sensitized subjects. Based on our preclinical results DermAll ASIT should balance the allergen-induced pathogenic $\mathrm{T}_{\mathrm{H}}$ 2-polarized immune responses and prevent the development of allergic symptoms. We found that immediately after allergen exposure DermAll ASIT was less effective in reducing allergic symptoms but it promoted significantly the immunological recovery. Based on our preclinical data, we recommend starting DermAll ASIT in an allergen-free period to achieve clinically significant improvement during the following allergen exposure. However, we expect a faster recovery in the absence of safety problems when we continue DermAll ASIT during the next period of allergen exposures because we keep balancing the allergen-specific immune responses even during symptomatic period. DermAll ASIT could be also efficient to prevent the disease progression for example from allergic rhinitis to allergic asthma. We believe that our novel DermAll immunotherapeutic approach would be safe and effective not only for the treatment of AR, but also for other IgE-mediated diseases such as venom or food allergy.

\section{Acknowledgments}

We thank Judit Baunoch, Sztanyik Endréné and Tanácsné Bajkán Andrea for their excellent technical assistance.

\section{References}

1. Compalati E, Penagos M, Henley K, Canonica GW. Allergy Prevalence Survey by the World Allergy Organization. $J$ World Allergy Org 2007; 19:82-90

2. Pawankar R, Canonica GW, Holgate ST, Lockey RF. WAO white book on allergy. World Allergy Organisation; 2011:1-24.

3. Bousquet J, Khaltaev N, Cruz AA, Denburg J, Fokkens WJ, Togias A, et al. Allergic Rhinitis and its Impact on Asthma (ARIA) 2008 update (in collaboration with the World Health Organization, GA(2)LEN and AllerGen). Allergy 2008;63(Suppl 86):8-160.

4. Meltzer EO. Quality of life in adults and children with allergic rhinitis. J Allergy Clin Immunol 2001;108:S45-53.

5. Plaut M, Valentine MD. Clinical practice. Allergic rhinitis. N Engl J Med 2005;353:1934-44.
6. Bousquet J, Lockey R, Malling HJ. Allergen immunotherapy: therapeutic vaccines for allergic diseases. A WHO position paper. J Allergy Clin Immunol 1998;102:558-62.

7. Zuberbier T, Bachert C, Bousquet PJ, Passalacqua G, Walter CG, Merk $\mathrm{H}$, et al. GA(2) LEN/EAACI pocket guide for allergen-specific immunotherapy for allergic rhinitis and asthma. Allergy 2010;65: 1525-30.

8. Brozek JL, Bousquet J, Baena-Cagnani CE, Bonini S, Canonica GW, Casale TB, et al. Allergic Rhinitis and its Impact on Asthma (ARIA) guidelines: 2010 revision. J Allergy Clin Immunol 2010;126:466-76.

9. Bousquet J, Schunemann HJ, Samolinski B, Demoly P, Baena-Cagnani $\mathrm{CE}$, Bachert $\mathrm{C}$, et al. Allergic Rhinitis and its Impact on Asthma (ARIA): Achievements in 10 years and future needs. J Allergy Clin Immunol 2012;130:1049-62.

10. Noon L. Prophylactic inoculation against hay fever. Lancet 1911;177: 1572-3.

11. Larche M, Akdis CA, Valenta R. Immunological mechanisms of allergen-specific immunotherapy. Nat Rev Immunol 2006;6:761-71.

12. Focke-Tejkl M, Valenta R. Safety of engineered allergen-specific immunotherapy vaccines. Curr Opin Allergy Clin Immunol 2012;12: 555-83.

13. Akdis M, Akdis CA. Therapeutic manipulation of immune tolerance in allergic disease. Nat Rev Drug Discov 2009;8:645-60.

14. Frew AJ. Allergen immunotherapy. J Allergy Clin Immunol 2010;125: S306-13.

15. Senn G, Crivellaro M, Schiappoli M, Passalacqua G, Canonica GW. The safety of allergen immunotherapy. Allergy Clin Immunol Int: $J$ World Allergy Org 2007;19:95-9.

16. Senti G, von Moos S, Kundig TM. Epicutaneous allergen administration: is this the future of allergen-specific immunotherapy? Allergy 2011;66:798-809.

17. Pautrizel R, Cabanieu G, Bricaud H, Broustet P. Allergenic group specificity and therapeutic consequences in asthma; specific desensitization method by epicutaneous route. Sem Hop 1957;33:1394-403.

18. Blamoutier P, Blamoutier J, Guibert L. Treatment of pollinosis with pollen extracts by the method of cutaneous quadrille ruling. Presse Med 1959;67:2299-301.

19. Senti G, Graf N, Haug S, Ruedi N, von Moos S, Sonderegger T, et al. Epicutaneous allergen administration as a novel method of allergenspecific immunotherapy. J Allergy Clin Immunol 2009;124:997-1002.

20. Mondoulet L, Dioszeghy V, Ligouis M, Dhelft V, Dupont C, Benhamou $\mathrm{PH}$. Epicutaneous immunotherapy on intact skin using a new delivery system in a murine model of allergy. Clin Exp Allergy 2010;40:659-67.

21. Senti G, von Moos S, Tay F, Graf N, Sonderegger T, Johansen P, et al. Epicutaneous allergen-specific immunotherapy ameliorates grass polleninduced rhinoconjunctivitis: A double-blind, placebo-controlled dose escalation study. J Allergy Clin Immunol 2012;129:128-35.

22. Broos S, Lundberg K, Akagi T, Kadowaki K, Akashi M, Greiff L, et al. Immunomodulatory nanoparticles as adjuvants and allergen-delivery system to human dendritic cells: Implications for specific immunotherapy. Vaccine 2010;28:5075-85.

23. Martinez-Gomez JM, Johansen P, Erdmann I, Senti G, Crameri R, Kundig TM. Intralymphatic injections as a new administration route for allergenspecific immunotherapy. Int Arch Allergy Immunol 2009;150:59-65.

24. Lisziewicz J, Trocio J, Whitman L, Varga G, Xu J, Bakare N, et al. DermaVir: a novel topical vaccine for HIV/AIDS. J Invest Dermatol 2005;124:160-9.

25. Lisziewicz J, Toke ER. Nanomedicine applications towards the cure of HIV. Nanomedicine 2012.

26. Lisziewicz J, Bakare N, Calarota SA, Banhegyi D, Szlavik J, Ujhelyi E, et al. Single DermaVir Immunization: Dose-Dependent Expansion of Precursor/Memory T Cells against All HIV Antigens in HIV-1 Infected Individuals. PLoS One 2012; 7:e35416.

27. Mine Y, Yang M. Epitope characterization of ovalbumin in BALB/c mice using different entry routes. Biochim Biophys Acta 2007;1774: 200-12. 
28. Miyahara S, Miyahara N, Takeda K, Joetham A, Gelfand EW. Physiologic assessment of allergic rhinitis in mice: role of the high-affinity IgE receptor (FcepsilonRI). J Allergy Clin Immunol 2005;116:1020-7.

29. Spies B, Hochrein H, Vabulas M, Huster K, Busch DH, Schmitz F, et al. Vaccination with plasmid DNA activates dendritic cells via Toll-like receptor 9 (TLR9) but functions in TLR9-deficient mice. $J$ Immunol 2003;171:5908-12.

30. Lorincz O, Toke ER, Somogyi E, Horkay F, Chandran PL, Douglas JF, et al. Structure and biological activity of pathogen-like synthetic nanomedicines. Nanomedicine 2012;8:497-506.

31. Lisziewicz J, Trocio J, Xu J, Whitman L, Ryder A, Bakare N, et al. Control of viral rebound through therapeutic immunization with DermaVir. AIDS 2005;19:35-43.
32. Ito K, Ishimaru T, Kimura F, Matsudomi N. Importance of N-glycosylation positioning for secretion and folding of ovalbumin. Biochem Biophys Res Commun 2007;361:725-31.

33. Van Ree R, Van Leeuwen WA, Dieges PH, van Wijk RG, De Jong N, Brewczyski PZ, et al. Measurement of IgE antibodies against purified grass pollen allergens (Lol p 1, 2, 3 and 5) during immunotherapy. Clin Exp Allergy 1997;27:68-74.

34. Gleich GJ, Zimmermann EM, Henderson LL, Yunginger JW. Effect of immunotherapy on immunoglobulin $\mathrm{E}$ and immunoglobulin $\mathrm{G}$ antibodies to ragweed antigens: a six-year prospective study. $J$ Allergy Clin Immunol 1982;70:261-71.

35. Akdis CA, Akdis M. Mechanisms of allergen-specific immunotherapy. J Allergy Clin Immunol 2011;127:18-27. 